\title{
DIGITAL MODULATION CLASSIFICATION USING POWER MOMENT MATRICES
}

\author{
Alfred O. Hero III, Hafez Hadinejad-Mahram
}

\author{
4229 EECS, University of Michigan, Ann Arbor, MI 48109
}

\begin{abstract}
With the rising number of modulation types used in multi-user and multi-service digital communication systems, the need to find efficient methods for their discrimination in the presence of noise has become increasingly important. Here, we present a new approach based on a recently developed pattern recognition method previously applied to word spotting problems in binary images [1]. In this approach, a large number of spatial moments are arranged in a symmetric positive definite matrix for which eigendecomposition and noise subspace processing methods can be applied. The resultant denoised moment matrix has entries which are used in place of the raw moments for improved pattern classification. In this paper, we generalize the moment matrix technique to grey scale images and apply the technique to discrimination between $\mathrm{M}$-ary PSK and QAM constellations in signal space. Invariance to unknown phase angle and signal amplitude is achieved by representing the in-phase and quadrature components of the signal in the complex plane, and computing joint moments of normalized magnitude and phase components.
\end{abstract}

\section{INTRODUCTION}

Automated detection and classification of modulation type is an important problem arising in non-cooperative communications environments, surveillance, and multi-user networks. A wide variety of techniques have been proposed for constant and non-constant envelope signals such as M-ary PSK, FSK and QAM including: zero crossing analysis [3], likelihood ratio approximation [7, 6], wavelet expansions [5], higher order correlations [2], and moment methods [8].

This paper describes an alternative moment-based method which generalizes the approach of [8], that was based solely on the eighth order phase moment, in two ways: 1) we use linear combinations of a large number of different orders (more than 100) of joint phase and magnitude moments, 2) we give a denoising procedure for extracting signal-alone joint moments from noise contaiminated measurements. Specifically, we propose a method for classification of modulation types for M-ary PSK, FSK and QAM using eigendecompositions of moment matrices, developed in [1], which are highly resistant to additive noise, unknown phase angle and unknown magnitude.

The paper is organized as follows. First, we introduce a set of rotation and scale moment invariants by mapping signal space to a polar representation of the IQ plane centered at the origin. After giving a brief review of spatial power moments and power moment matrices [1], we demonstrate how to process noise contaminated bandpass signals to effectively separate signal components from

\footnotetext{
${ }^{1}$ This research was supported in part by the Army Research Office under grant
} DAAH04-96-1-0337. noise background via eigendecomposition. This requires extending the power moment matrix introduced in [1] to gray scale images, which is achieved by representing the image by three coordinates ( 2 spatial and one grey scale) and computing a larger matrix of higher order pairwise correlations. To illustrate the methodology, we consider discrimination of noise contaminated 4-ary PSK and QAM signal constellations with different noise variances.

\section{MOMENT INVARIANTS IN THE PLANE}

Similar to the definition of moments from classical mechanics, the $(p+q)$ th order spatial moment of an image irradiance destribution $f(x, y)$ is defined by

$$
m^{p, q}=\iint_{-\infty}^{\infty} x^{p} y^{q} f(x, y) d x d y
$$

Li [4] showed that certain combinations of these moments are invariant with respect to translation, scale and rotation. These invariances are, however, ill-suited for the modulation discrimination problem. For example, in case of 4-PSK, there are four points symmetrically centered at the origin of the coordinates on the complex plane whose locations are determined by $A e^{j \theta_{i}}, i=1,2,3,4$. Changes of $A$ and/or $\theta$ result in changes of the magnitude and/or rotation of the points. Therefore, invariance under translation is not useful. On the other hand, Li's scale invariance is only valid for changes of the image size provided the image contains a finite area. In case of modulation patterns, the images consist of points and have, therefore, an area of zero. Thus this invariance is not useful either. Finally, the classical moment invariants do not provide invariance with respect to changes of the magnitude in polar coordinates. In the following we will derive a new set of moment invariants that will satisfy both of the invariance requirements.

Because of the special structure of IQ plane containing in-phase and quadrature components, we represent the measurements in polar coordinates of the complex plane. Define moments of phasemagnitude polar representation of the IQ image

$$
\begin{aligned}
m_{\boldsymbol{r}, \boldsymbol{\theta}}^{p, \boldsymbol{q}} & =E\left[\left(\boldsymbol{r} / r_{0}\right)^{p} e^{j \boldsymbol{\theta} q}\right]=\int_{0}^{2 \pi} \int_{0}^{\infty}\left(r / r_{0}\right)^{p} e^{j \theta q} f(r, \theta) d r d \theta \\
& =\sum_{i=0}^{n}\left(r_{i} / r_{0}\right)^{p} e^{j \theta_{i} q}
\end{aligned}
$$

where $r_{0}=\frac{1}{n} \sum_{i=0}^{n} r_{i}$ is the arithmetic mean of the magnitudes. Dividing $r$ by $r_{0}$ provides the invariance under magnitude changes as the following shows:

$$
m_{r^{\prime}, \theta}^{p, q}=E\left[\left(\boldsymbol{r}^{\prime} / r_{0}^{\prime}\right)^{p} e^{j \theta q}\right]=\sum_{i=0}^{n}\left(r_{i}^{\prime} / r_{0}^{\prime}\right)^{p} e^{j \theta_{i} q}
$$


where $r_{i}^{\prime}=c r_{i}$. Therefore, $r_{0}^{\prime}$ results in:

$$
r_{0}^{\prime}=\frac{1}{n} \sum_{i=0}^{n} r_{i}^{\prime}=\frac{1}{n} \sum_{i=0}^{n} c r_{i}=c r_{0}
$$

implying that $m_{r^{\prime}, \boldsymbol{\theta}}^{p, q}=m_{r, \theta}^{p, q}$. In order to make these moments rotation invariant, we use the magnitude squared of the complex moments:

$$
\begin{aligned}
\left|m_{\boldsymbol{r}, \boldsymbol{\theta}+\Delta}^{p, q}\right|^{2} & =\left|E\left[\left(\boldsymbol{r} / r_{0}\right)^{p} e^{j \theta q}\right]\right|^{2}= \\
& =\left|E\left[\left(\boldsymbol{r} / r_{0}\right)^{p} e^{j(\boldsymbol{\theta}+\Delta) q}\right]\right|^{2} \\
& =\left|E\left[\left(\boldsymbol{r} / r_{0}\right)^{p} e^{j \boldsymbol{\theta} q} e^{j \Delta q}\right]\right|^{2}=\left|m_{\boldsymbol{r}, \boldsymbol{\theta}}^{p, q}\right|^{2}
\end{aligned}
$$

\section{SPATIAL POWER MOMENTS}

Let $f=f(\underline{x})$ be the density function of a random point $\underline{x}$ in an $n$ dimensional space $\mathbf{R}^{n}$. It is well known that $f(\underline{x})$ can be specified by its set of higher order moments of mixed orders. Define the gray scale IQ plane representation by the three variables $(\underline{x}=(r, \theta, z))$, where $r, \theta$ are polar coordinates of each pixel and $z$ is the gray level of this pixel. Observe that this involves an extra variable $z$ as compared to the case of binary images treated in [1]. We define spatial power moments (PM) $\mu_{r, \theta}(p, q, s)$ of (integer) order $p, q, s$ of positive integer random variables $r, \theta$ and $z$ as:

$$
\mu_{r, \theta}(p, q, s)=E\left[\boldsymbol{r}^{p} e^{j \theta q} \boldsymbol{z}^{s}\right]
$$

It is well known that the set of $L^{3}$ power moments $\left\{\mu_{r, \theta}(p, q, s)\right\}_{p, q, s=1}^{L}$ completely characterizes the density in the limit as $L \rightarrow \infty$. However, in many applications the pairwise higher order moments of the form $E\left[\boldsymbol{r}^{p} \boldsymbol{\theta}^{q}\right], E\left[\boldsymbol{\theta}^{q} \boldsymbol{z}^{s}\right]$ and $E\left[\boldsymbol{r}^{p} \boldsymbol{z}^{s}\right]$ are sufficient to perform pattern discrimination whereby a significant part of the time consuming computation can be reduced.

\section{POSITIVE DEFINITE POWER MOMENT MATRICES}

The $(3 L+1) \times(3 L+1)$ power moment matrix (PMM) for a three dimensional random vector $(\boldsymbol{r}, \boldsymbol{\theta}, \boldsymbol{z})$ that contains only the pairwise higher order moments, as introduced in the last section, is defined as the expectation over $\boldsymbol{r}, \boldsymbol{\theta}, \boldsymbol{z}$ of the dyadic outer product of $\underline{U U^{*}}$ where $\underline{U}=$ $\left[1, \boldsymbol{r}, \boldsymbol{r}^{2}, \ldots, \boldsymbol{r}^{L}, e^{j \boldsymbol{\theta}}, e^{j \mathbf{2 \theta}}, \ldots, e^{j L \boldsymbol{\theta}}, \boldsymbol{z}, \boldsymbol{z}^{2}, \ldots, \boldsymbol{z}^{L}\right]^{T}$, or more explicitly it is the expectation over

$$
\left[\begin{array}{cccccccccc}
1 & \boldsymbol{r} & \ldots & \boldsymbol{r}^{L} & e^{-j \boldsymbol{\theta}} & \ldots & e^{-j L \boldsymbol{\theta}} & \boldsymbol{z} & \ldots & \boldsymbol{z}^{L} \\
\boldsymbol{r} & \boldsymbol{r}^{2} & \ldots & \boldsymbol{r}^{L+1} & \boldsymbol{r} e^{-j \boldsymbol{\theta}} & \ldots & \boldsymbol{r} e^{-j L \boldsymbol{\theta}} & \boldsymbol{r} \boldsymbol{z} & \ldots & \boldsymbol{r} \boldsymbol{z}^{L} \\
\vdots & \ddots & \ddots & \vdots & \vdots & \ddots & \vdots & \vdots & \ddots & \vdots \\
\boldsymbol{r}^{L} & \boldsymbol{r}^{L+1} & \ldots & \boldsymbol{r}^{2 L} & \boldsymbol{r}^{L} e^{-j \boldsymbol{\theta}} & \ldots \boldsymbol{r}^{L} e^{-j L \boldsymbol{\theta}} & \boldsymbol{r}^{L} \boldsymbol{z} & \ldots & \boldsymbol{r}^{L} \boldsymbol{z}^{L} \\
e^{j \boldsymbol{\theta}} & \boldsymbol{r} e^{j \boldsymbol{\theta}} & \ldots & \boldsymbol{r}^{L} e^{j \boldsymbol{\theta}} & 1 & \ldots & e^{j(1-L) \boldsymbol{\theta}} & \boldsymbol{z} e^{j \boldsymbol{\theta}} & \ldots & \boldsymbol{z}^{L} e^{j \boldsymbol{\theta}} \\
\vdots & \ddots & \ddots & \vdots & \vdots & \ddots & \vdots & \vdots & \ddots & \vdots \\
e^{j L \boldsymbol{\theta}} \boldsymbol{r} e^{j L \boldsymbol{\theta}} & \ldots & \boldsymbol{r}^{L} e^{j L \boldsymbol{\theta}} & e^{j(L-1) \boldsymbol{\theta}} & \ldots & 1 & \boldsymbol{z} e^{j L \boldsymbol{\theta}} & \ldots \boldsymbol{z}^{L} e^{j L \boldsymbol{\theta}} \\
& & & & & & & & & \\
\boldsymbol{z} & \boldsymbol{r} \boldsymbol{z} & \ldots & \boldsymbol{r}^{L} \boldsymbol{z} & \boldsymbol{z} e^{-j \boldsymbol{\theta}} & \ldots & \boldsymbol{z} e^{-j L \boldsymbol{\theta}} & \boldsymbol{z}^{2} & \ldots & \boldsymbol{z}^{L+1} \\
\vdots & \ddots & \ddots & \vdots & \vdots & \ddots & \vdots & \vdots & \ddots & \vdots \\
\boldsymbol{z}^{L} & \boldsymbol{r} \boldsymbol{z}^{L} & \ldots & \boldsymbol{r}^{L} \boldsymbol{z}^{2 L} & \boldsymbol{z}^{L} e^{-j \boldsymbol{\theta}} & \ldots \boldsymbol{z}^{L} e^{-j L \boldsymbol{\theta}} & \boldsymbol{z}^{L+1} & \ldots & \boldsymbol{z}^{2 L}
\end{array}\right]
$$

This moment matrix is obviously non-negative definite. However, the moment matrix we will be using consists of the magnitude squared of the moments and is created by an element by element multiplication of this matrix with its conjugate. It can easily be shown that this product is also non-negative definite which is a prerequisite for our following considerations.

\section{DENOISING VIA EIGENDECOMPOSITION}

Let $\mathbf{M}, \mathbf{M}_{s}$, and $\mathbf{M}_{n}$ denote the signal plus noise, signal alone, and noise alone moment matrices). Using the mixture model gives:

$$
\mathbf{M}=\beta \mathbf{M}_{s}+(1-\beta) \mathbf{M}_{n}
$$

Now let $\mathbf{C}$ be the known Cholesky factor of the positive definite noise-alone moment matrix $\mathbf{M}_{n}$, i.e. $\mathbf{M}_{n}=\mathbf{C C}^{T}$, and define $\tilde{\mathbf{M}}=$ $\mathbf{C}^{-1} \mathbf{M C}^{-T}$. Then we have:

$$
\tilde{\mathbf{M}}=\beta \tilde{\mathbf{M}}_{s}+(1-\beta) \mathbf{I}
$$

where $\mathbf{I}$ is the $(3 L+1) \times(3 L+1)$ identity matrix, and $\tilde{\mathbf{M}}_{s}=$ $\mathbf{C}^{-1} \mathbf{M C}^{-T}$ is the whitened moment matrix of the signal pattern. Consider the eigendecomposition:

$$
\tilde{\mathbf{M}}=\sum_{i=1}^{2 p+1} \gamma_{i} \xi_{i} \underline{\xi}_{i}^{T}
$$

where $\gamma_{i}$ and $\underline{\xi}_{i}$ are eigenvalues (rank ordered) and eigenvectors. Then the eigenvectors of $\tilde{\mathbf{M}}$ and $\tilde{\mathbf{M}}_{s}$ are identical, and from (1) we have

$$
\tilde{\mathbf{M}}=\sum_{i=1}^{q}\left[\beta \gamma_{i}^{s}+(1-\beta)\right] \underline{\xi}_{i} \underline{\xi}_{i}^{T}+(1-\beta) \sum_{i=q+1}^{2 p+1} \xi_{i} \xi_{i}^{T}
$$

where $\left\{\gamma_{i}^{s}\right\}_{i=1}^{q}$ are the non-zero eigenvalues of $\tilde{\mathbf{M}}_{s}$. Thus only the $q$ largest eigenvalues $\gamma_{i}=\beta \gamma_{i}^{s}+(1-\beta)$ of $\tilde{\mathbf{M}}$ are related to the signal pattern and the rest are pure noise eigenvalues $\{(1-$ $\beta) \ldots,(1-\beta)\}$. In particular, $\tilde{\mathbf{M}}_{s}$ can be exactly recovered from the eigendecomposition of $\tilde{\mathbf{M}}$ via

$$
\tilde{\mathbf{M}}_{s}=\frac{1}{\beta} \sum_{i=1}^{q}\left[\gamma_{i}-(1-\beta)\right] \underline{\xi}_{i} \underline{\xi}_{i}^{T}
$$

Since the $(1,1)$ element of $\mathbf{M}_{s}$ is equal to 1 , and $\underline{e}_{1}^{T} \mathbf{C} \underline{\xi}_{i}=\underline{e}_{1}^{T} \underline{\xi}_{i}=$ $\xi_{i 1}$,

$$
\beta=\frac{\sum_{i=1}^{q}\left[\gamma_{i}-\left|\xi_{i 1}\right|^{2}\right]}{1-\sum_{i=1}^{q}\left|\xi_{i 1}\right|^{2}}
$$

\section{SCALING THE POWER MOMENT MATRIX}

When a variate, e.g. $r$, is contained in the interval $\left[r_{\min }, r_{\max }\right]$, we can improve the conditioning of $\mathbf{C}$ by centering and scaling $r$ into the interval $[-1,1]$. This is done by making the transformation $\tilde{r}=$ $\frac{r-\left(r_{\min }+r_{\max }\right) / 2}{\left(r_{\max }-r_{\min }\right) / 2}$. The same transformation must be accomplished for $\theta$ and $z$ in order to reach the best stability behavior. 


\section{EXAMPLES}

In this section we will illustrate the moment matrix discrimination technique for two different noise contaminated digital modulation types: 4-PSK and 4-QAM with similar signal constellations. The IQ detector block diagram is shown in Fig. 1. A signal is carrier modulated at carrier frequency $f_{o}$ to produce a transmitted signal $s(t)$. The transmitted signal passes through an ideal bandpass additive Gaussian noise channel producing a received bandpass signal $w(t)=s(t)+n(t)$. The in phase $\left(w_{I}\right)$ and quadrature $\left(w_{Q}\right)$ baseband components are detected separately by two orthogonal frequency mixers, each centered at frequency $w_{0}=2 \pi f_{o}$, each followed by an integrator. These components are each periodically sampled to produce the sequence of complex measurements $y_{I}\left(t_{i}\right)+j y_{Q}\left(t_{i}\right), i=1, \ldots, N$, which can be represented as a point cloud of $N$ points in the complex (IQ) plane, inhabited by the complex signal constellation. For the experiments reported here $N=25$. It is in the IQ plane that we implement the moment matrix techniques described in the previous sections. Note that the channel noise produces a spatial blurring of the signal constellation in the IQ plane, i.e. the noise in the IQ plane is not spatially homogeneous and is signal dependent. For the experiments below we fixed an assumed signal modulation (4-PSK at unit power) and noise variance $\left(\sigma^{2}=25\right)$ and generated the Cholesky factors of the associated moment matrix. This Cholesky factor was then used to "whiten" the empirically calculated moment matrices for each of the measured signal types. Note that in each of the experiments described below this whitening is mismatched to the actual noise distribution in the IQ plane. Nonetheless, we will show that an improvement in modulation discrimination results even with a moderate amount of mismatch.

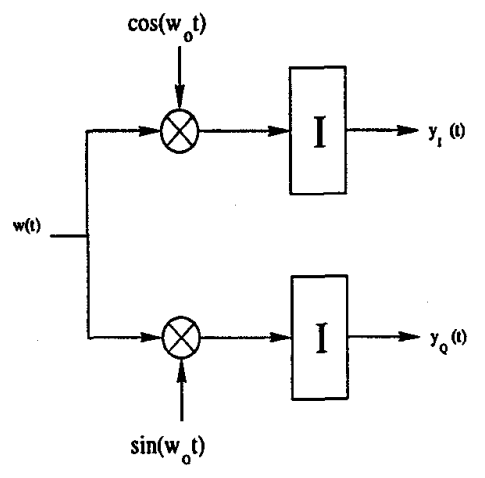

Figure 1: Coherent IQ receiver for bandpass carrier modulated signal $w(t)$ at carrier frequency $w_{o}=2 \pi f_{o}$.

Figures 2 and 3 illustrate the IQ images and moment matrices for the cases that $s(t)$ is a 4-PSK signal with noise power of $\sigma^{2}=5$, while Figs. 4 and 5 are for 4-QAM with $\sigma^{2}=5$ ). The first column of each of these figures are, from top to bottom: the signal alone represented in the IQ plane, the unwhitened power moment matrix of the signal, the whitened power moment matrix of the signal, and the assumed spatial distribution of the noisy measurements used to calculate the Cholesky factor of the moment matrix. The second column of these figures are, from top to bottom: the noise contaminated signal in the IQ plane, the unwhitened moment matrix estimated from the $N$ noisy measurements, the estimated whitened moment matrix, and the resultant cleaned power moment matrix after noise subspace processing.
By comparing the signal alone unwhitened moment matrix to that of the noise contaminated signal it is evident that the raw moments are quite sensitive to additive noise (compare left and right panels in the second rows of Figs. 2-5). On the other hand, by comparing the left and right panels in each of third rows of the figures, it is evident that the prewhitened moment matrices are much less sensitive to noise. This can be explained by the fact that, as the variables $\underline{x}=(r, \theta, z)$ are prenormalized to the interval $[-1,1]$, the higher order moments $E\left[r^{p} \theta^{q}\right], E\left[\theta^{q} z^{s}\right]$ and $E\left[r^{p} z^{s}\right]$ are exponentially decreasing to zero as $p, q, s$ become large. The prewhitening of the moment matrix via Cholesky decomposition rescales all of these moments to produce entries of comparable magnitudes and thus all high and low order moments are put on equal footing. This matrix rescaling can be interpreted as a generalization of variance normalization, such as those used to produce correlation coefficients and spectral coherence functions, which have been widely used to study dependencies in two or more random variables of greatly different average magnitudes.

It is noteworthy that even with noise mismatch the whitened and cleaned moment matrices provide a stable representation of any specified noise'contaminated modulation (note similarity of 3rd rows of Figs. 2 and 3 for 4-PSK with two different received noise powers) while they provide a good degree of discrimination power between different modulation formats (note dissimilarity of 3rd rows of Figs. 2 and 4). Note also that, by construction, the moment representations are invariant to rotation and scale of the signal constellation due to unknown carrier phase angle or signal amplitude. Figure 5 shows the moment matrices for the case of 4-QAM where the exact constellation noise distribution, shown in the lower left hand panel, is used to construct the Cholesky decomposition. We are currently running extensive Monte-Carlo simulations to quantify the improved discrimination probabilities suggested by the experiments reported here.

\section{REFERENCES}

[1] B. F. Beidas and C. L. Weber, "Higher-order correlation-based approach to modulation classification of digitally frequency-modulated signals," IEEE Journal on Selected Areas in Communications, vol. 13, pp. 89-101, Jan. 1995.

[2] A. O. Hero, J. O' Neill, and W. J. Williams, "Moment matrices for recognition of spatial pattern in noisy images," IEEE Int. Conf. on Image Processing, Okt. 1997.

[3] S.-L. Hsue and S. Soliman, "Automatic modulation recognition of digitally modulated signals," in Proceedings of MILCOM, pp. 645647, Boston, MA, 1989.

[4] Y. Li., "Reforming the theory of invariant moments for pattern ricognition," Pattern Recognition, no. 7, pp. 723-730, 1992.

[5] Y.-C. Lin and C.-C. J. Kuo, "Practical psk modulation classifier using wavelets," in Proceedings of SPIE, pp. 492-503, Bellingham, WA, 1995.

[6] C. Long, K. Chugg, and A. Polydoros, "Further results in likelihood classification of qam signals," in Proceedings of MILCOM, pp. 57-61, Long Branch, NJ, 1994.

[7] A. Polydoros and K. Kim, "On the detection and classification of quadrature digital modulations in broad-band noise," IEEE Trans. on Communications, vol. 38, pp. 1199-1211, Aug. 1990.

[8] S. S. Soliman and S.-Z. Hsue, "Signal classification using statistical moments," IEEE Trans. on Communications, vol. 40, pp. 908-916, May. 1992. 


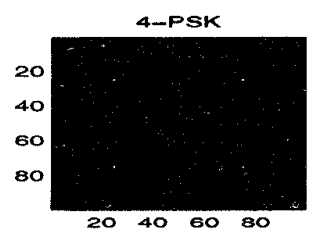

20406080

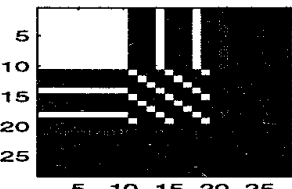

$10 \quad 152025$
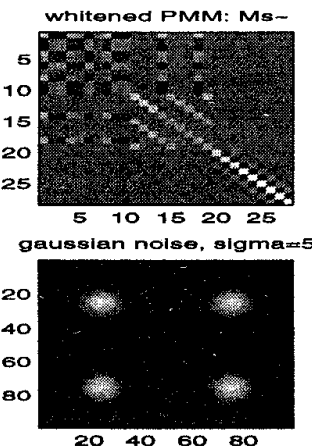

$20 \quad 40 \quad 60 \quad 80$

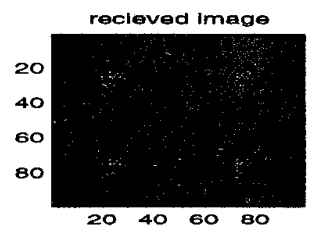

hitened PMM: $M \quad 4=$

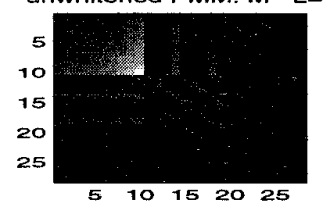

whitened PMM: M-
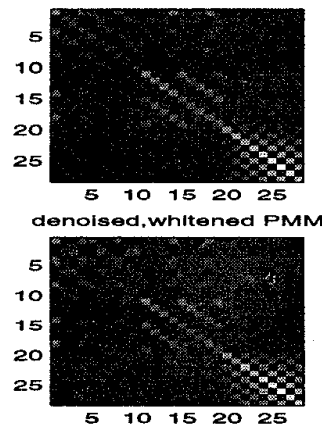

$5 \quad 10 \quad 15 \quad 20 \quad 25$

Figure 2: First row: signal alone (left) and noise contaminated IQ images for 4-PSK, second row: unwhitened PMMs, third row: whitened PMMs, fourth row: spatial distributuion of the noise(left) and the denoised, whitened PMM.
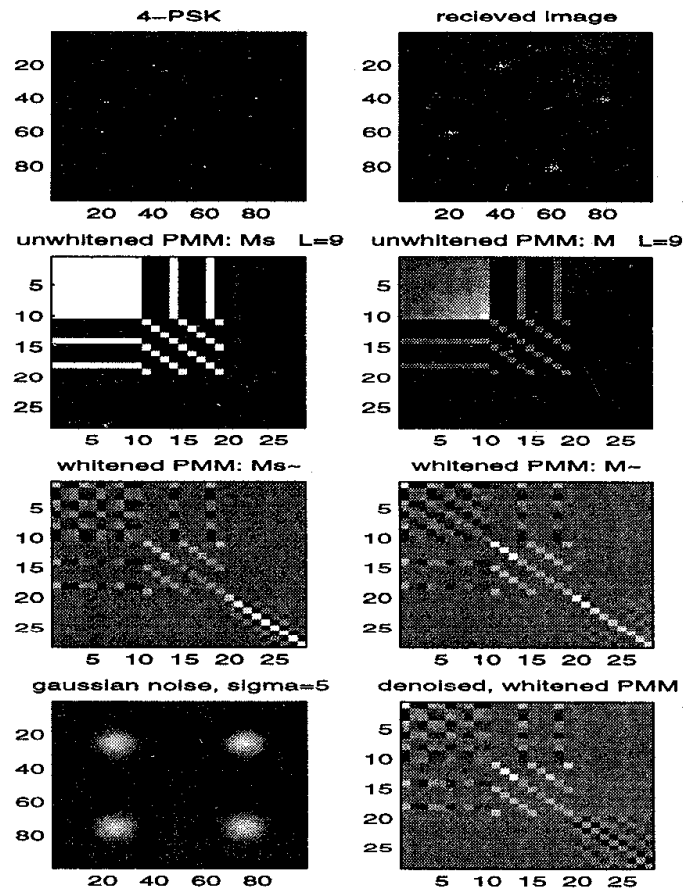

Figure 3: The order of the panels is the same as in figure 2. Note that there is now a mismatch between the noise and the the actual spatial destribution of the received signal. Note further the rotation invariance of PMM.
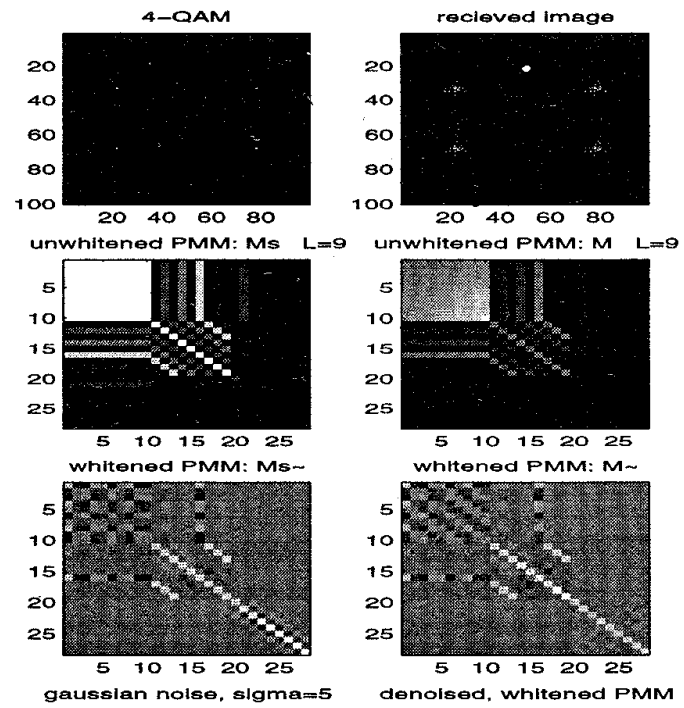

$\begin{array}{ccccc}5 & 10 & 15 & 20 & 25 \\ \text { whitened } & \text { PMM: } & \text { M } ~\end{array}$
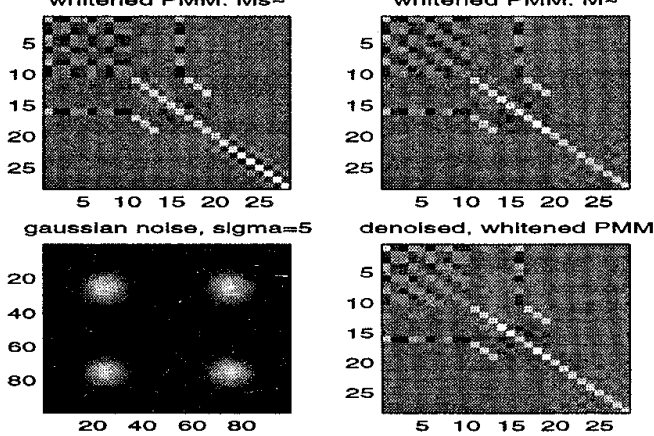

Figure 4: First row: signal alone (left) and noise contaminated IQ images for 4-QAM, second row: unwhitened PMMs, third row: whitened PMMs, fourth row: spatial distributuion of the noise(left) and the denoised, whitened PMM. Note that the wrong cholesky factor is used, i.e. the cholesky factor corresponding to 4-PSK.
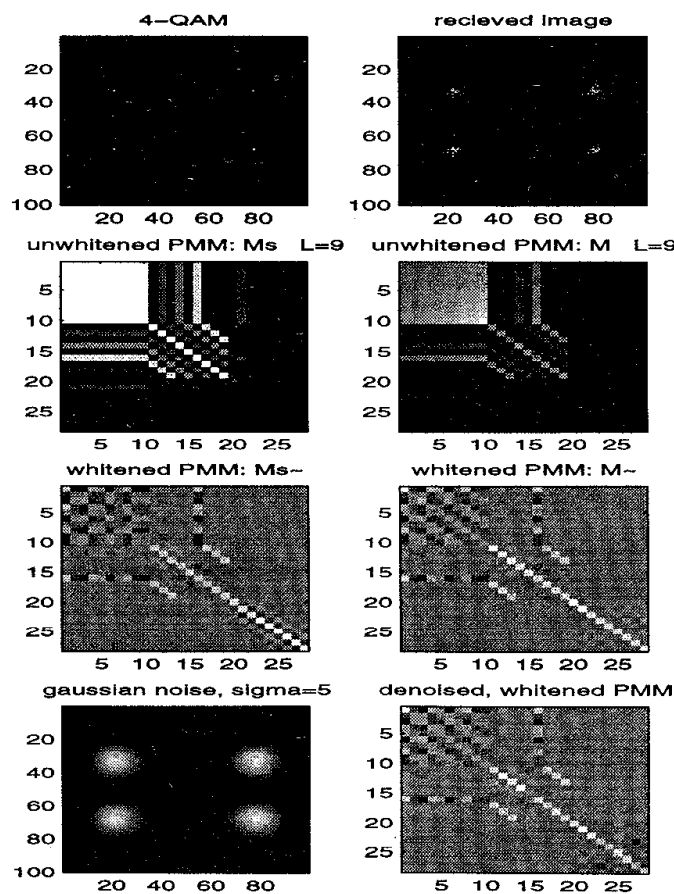

Figure 5: The order of the panels is the same as in figure 4 . There is hardly any difference between the moment matrices of figure 4 and the moment matrices in this figure (with the correct cholesky factor). 